\title{
An Intelligent Remote Monitoring System for Total Knee Arthroplasty Patients
}

Yunus Msayib $^{1}$, Patrick Gaydecki ${ }^{2}$, Michael Callaghan ${ }^{3}$, Nicola Dale $^{4}$, and Sheheera Ismail ${ }^{2}$

${ }^{1}$ Institute of Biomedical Engineering, Department of Engineering Science, University of Oxford, OX3 7DQ UK

${ }^{2}$ School of Electrical and Electronic Engineering, the University of Manchester, Manchester, M13 9PL, UK

${ }^{3}$ School of Translational Medicine, the University of Manchester, Manchester, M13 9PL, UK

${ }^{4}$ Arthritis Research UK Epidemiology Unit, Institute of Inflammation and Repair, the University of Manchester, Manchester, M13 9PL, UK

Declarations: The authors declare no conflict of interest. 


\begin{abstract}
For the first six weeks following total knee arthroplasty (TKA), a patient will attend an outpatient clinic typically seen twice weekly. Here, an exercise regime is performed and improvement assessed using a hand held goniometer that measures the maximum angle of knee flexion, an important metric of progress. Additionally a series of daily exercises is performed at home, recorded in a diary. This protocol has problems. Patients must attend the hospital with assistance since they are not permitted to drive for six weeks following the procedure; appointments are sometimes missed; there are occasionally not enough physiotherapy appointment available; furthermore, it is difficult to be sure that patients are compliant with their exercises at home. The economic and social costs are therefore significant both to the patient and the health service.

We describe here an automatic system that performs the monitoring of knee flexion within a domestic environment rather than in a hospital setting. It comprises a master and slave sensor unit that attach using Velcro straps to the thigh and shin above and below the operation wound. The patient performs the prescribed knee exercises whilst wearing the device, during which time it measures and records the angles of knee flexion. The device utilises the Global System for Mobile Communications (GSM) infrastructure to transmit data through the Internet to a secure hospitalbased server using an on-board GSM modem. The clinician is then able to view and interpret the information from any computer with internet access and the software. The system does not require the patient to possess a mobile telephone, a computer, or have internet access; the necessary communications technology is completely integrated into the device.
\end{abstract}




\section{Introduction}

In the USA there are approximately $581,000 \mathrm{TKA}^{1}$ procedures annually [3], with a $100 \%$ increase between 1997 and 2007 [3]. It is estimated that this figure will grow further by $340 \%$ over the next fifteen years [4]. This multi-fold increase will also happen in the UK, where the number of TKA procedures presently stands at more than 66,000 per annum [1]. This is due to the increased incidence of arthritis and related issues such as obesity and longevity. The increase in surgery will also mean greater demands on nursing and physiotherapy.

The current practice of clinic-based TKA rehabilitation appointments is demanding on both the resources of the health provider and on outpatients' time and near-term quality of life. This is instigated by the temporary handicap on patients' mobility following TKA, including their inability to drive for at least the first six post-operative weeks [5]. In the UK this leads to health service costs incurred through missed appointments and clinician time, as well as placing time and travel demands on the ambulance service, community carers, and relatives $[6,7]$.

Here is described a self-contained intelligent device for remote domiciliary monitoring of the amount of knee flexion following TKA. The remote flexion monitoring (RFM) system could be used by clinical physiotherapists assessing the recovery progress of TKA patients, thus largely eliminating the need for the traditional practice of hospital-based follow-up appointments and associated burdens.

\section{Burdens of Routine Outpatient Physiotherapy Appointments}

Presently, for the first six weeks following TKA, patients are seen twice-weekly in a physiotherapy outpatient department where they perform an exercise regime. Their progression or regression is assessed by clinicians using a standard hand-held goniometer which measures the angles of knee flexion and extension, and is an important and objective measure [8]. Additionally, TKA patients are prescribed an established routine of daily exercises including knee flexion and extension to be performed at their home in-between hospital physiotherapy appointments, and which are often recorded by the patient in a self-reported exercise diary. This protocol has a number of problems. Patients must attend the hospital with assistance since they are not permitted to drive for six weeks following the procedure; there are occasionally not enough physiotherapy appointments available; and owing largely to these difficulties, appointments are sometimes missed leading to wasted clinician time thus, within the UK, further straining NHS resources. Furthermore, due to well-

1 Total Knee Arthroplasty: surgical procedure to replace arthritic weight-bearing surfaces of the knee joint 
known problems associated with patients' exercise diaries [9-11], it is difficult to be sure that patients are compliant with their prescribed exercises.

The current practice of TKA post-operative follow-up appointments engenders the prolongation of hospital outpatient times, waiting lists, and physiotherapy appointment times, as well as placing significant demand on patients' time by way of travel issues. The annual UK clinical cost associated with the totality of the present system is $£ 23.3 \mathrm{M}$ (\$39.5 million USD) in outpatient appointments $[1,2]$.

\section{Benefits of Remote Flexion Monitoring}

Within the NHS and equivalent services across the world there has been a growing awareness of the advantages of remote healthcare monitoring. This has been facilitated in large measure by the ubiquity of the Internet, allowing data to be streamed from any location to any destination globally. Remote healthcare systems offer significant benefits in relation to social, environmental, and economic costs and compliance verification. Moreover, they allow both patients and clinical staff greater freedom with respect to time management and work optimisation [12]. The model of TKA is a relatively simple one compared to other complex pathologies and conditions such as stroke, obesity and weight loss and other orthopaedic operations. This is because progression and regression during the post-operative period is measured to a large extent by the degrees of knee flexion that a patient can attain. For physiotherapy these demands may be eased by the use of remote monitoring instead of the traditional practice of seeing patients in the hospital outpatients setting twice weekly. It is evident that the necessity and demand for such a system is significant and expanding rapidly.

Remote flexion monitoring has many advantages to offer over the traditional patient attendance system; in particular it benefits economically both the patient and the health service. Furthermore, it frees the clinician from the constraints of traditional consultation, and by automating the diarykeeping process through an intelligent device, it provides an objective measure of patient compliance.

\section{Materials and Methods}

The remote flexion monitoring system (RFM system) (Fig. 1) comprises three main components: an intelligent electronic remote flexion monitoring device (RFM device) that the patient wears at home to record exercise performance; a dedicated hospital-based secure server that receives, stores, and 
transmits the patient's exercise data; and a Java application (app) that the clinician uses to download, analyse, and index the patient's exercise data.

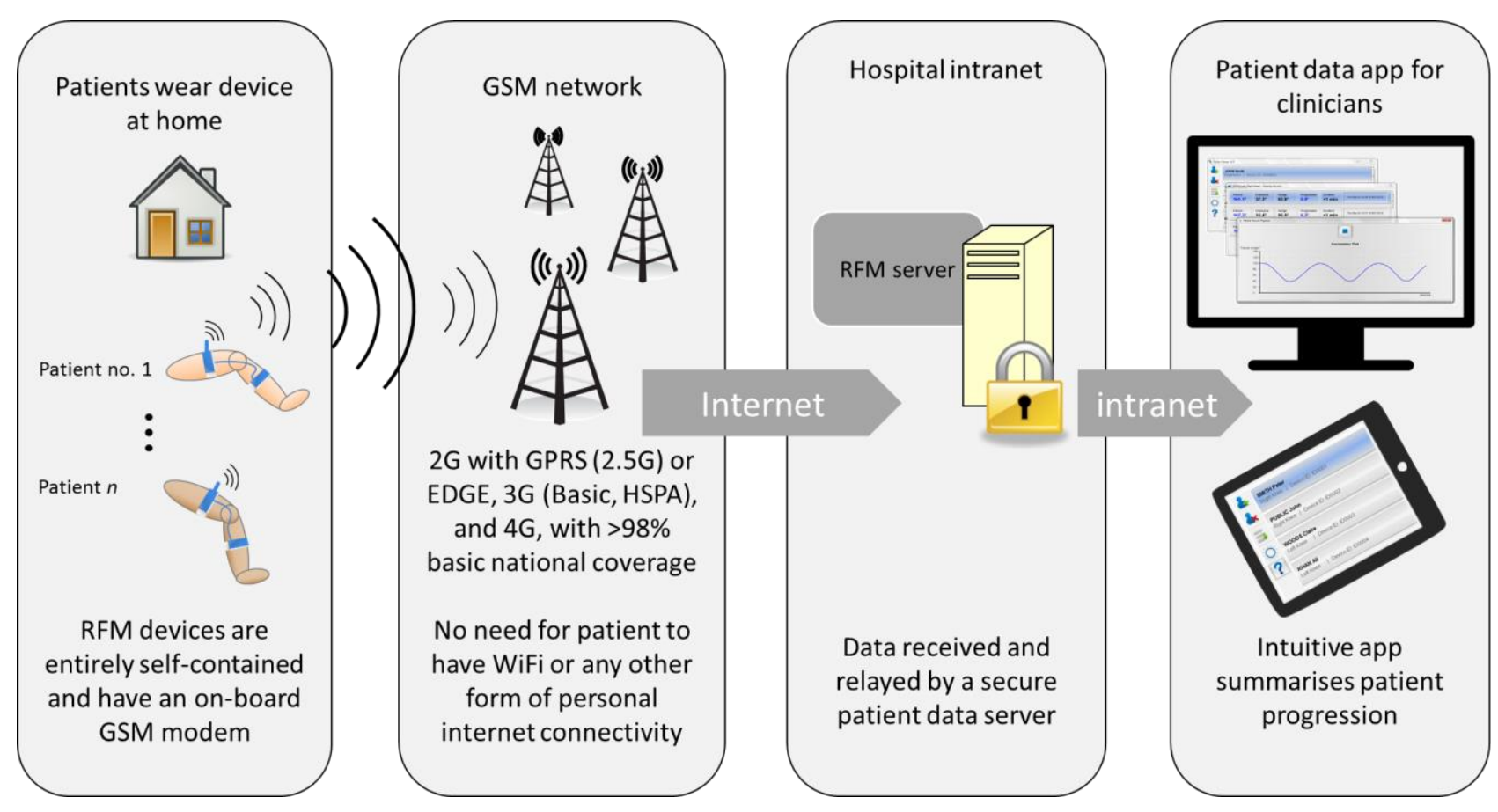

Fig. 1 Remote flexion monitoring system architecture: data flow from remotely-located patients to the clinician app is shown

With the average age of TKA patients being 69 years [1], convenience and ease of use are essential features of the monitoring device. The RFM device operates entirely autonomously within a domestic environment; it comprises a master and slave sensor unit that attach easily to the thigh and shin through the use of an adjustable Velcro straps on each unit. The patient performs the prescribed knee exercises whilst wearing the device, during which time the unit automatically measures and records the angles of knee flexion. Using its integrated modem, the device transmits the data via the $\mathrm{GSM}^{2}$ infrastructure and then to the Internet to a secure hospital-based server, requiring no patient interaction. It is important to emphasise that the patient does not require Wi-Fi or Internet access, nor indeed possess a computer or a mobile (cell) phone. All data are acquired and transmitted by the monitoring device. The clinician is then able to view and interpret the information from any computer with access to the Hospital's intranet through the use of a web-distributed analysis program. Not only can the clinician assess the progression or regression of the patient, since the data are date-time stamped and timed, it is also possible to determine the regularity with which the exercises are performed and how much time the patient sets aside for them.

\footnotetext{
${ }^{2}$ Global System for Mobile Communications: Global mobile communications standard that describes protocols for second generation $(2 \mathrm{G})$ digital cellular networks used by mobile phones
} 


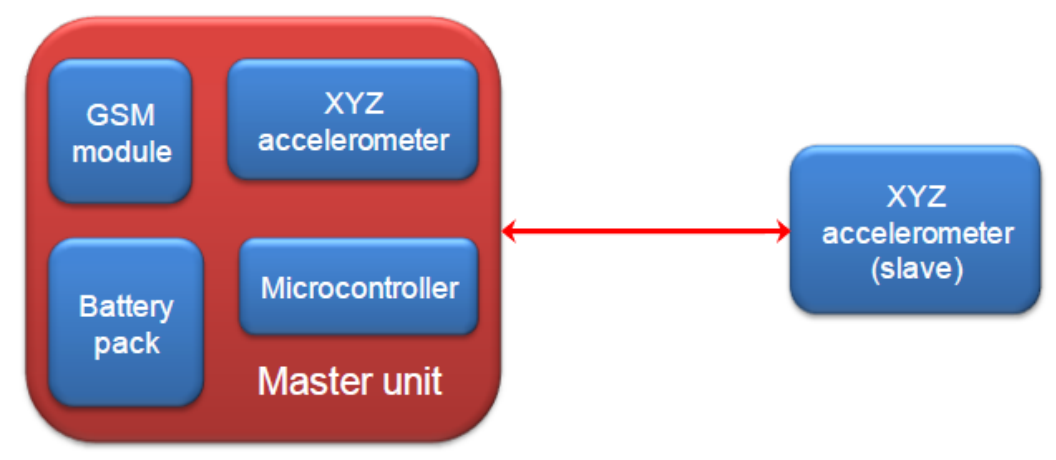

Fig. 2 Schematic depicting the main components of the RFM device electronics which comprise a master and slave unit

\section{RFM Device Electronics}

Device intelligence is realised through the use of a programmable 8-bit microcontroller which coordinates the operation of the monitoring device including the sensor angle electronics, the integrated GSM modem and power management facilities. This is shown schematically in Fig. 2, with a photograph of the master unit in Fig. 3.

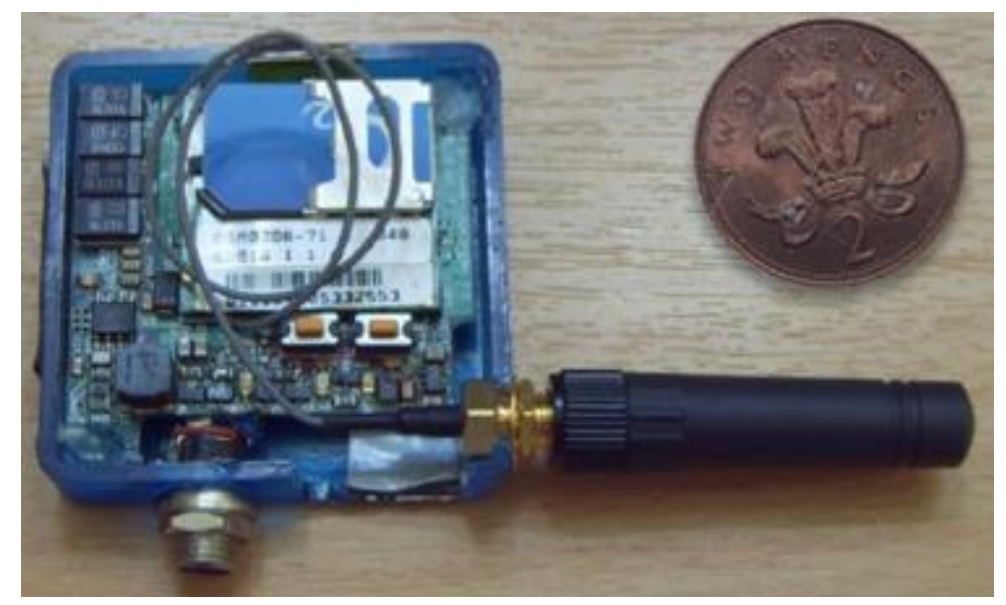

Fig. 3 RFM device master unit in which the 2.5G GSM modem is visible with a size comparison 


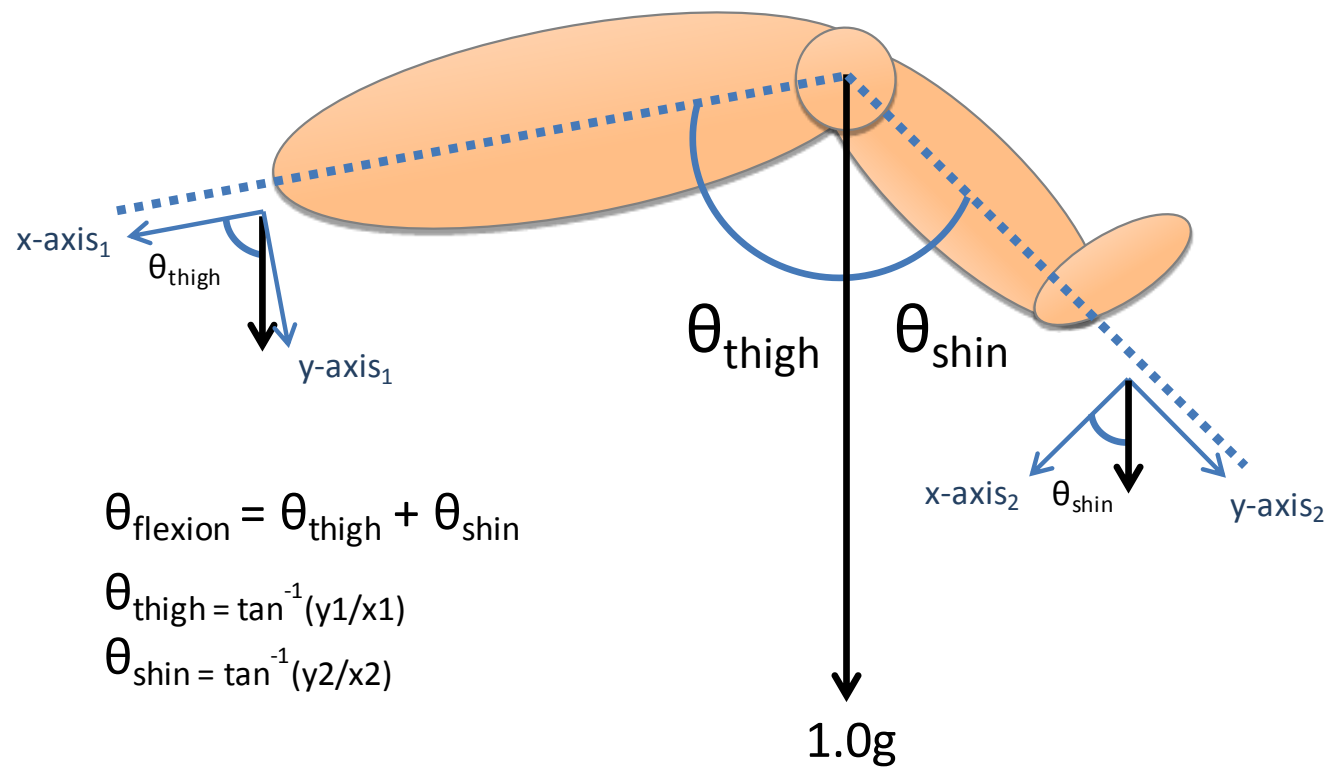

Fig. 4 Trigonometric identities used for calculating the angle of knee flexion using two independent accelerometers; one strapped to the thigh, and the second strapped to the shin

Each master and slave unit contains a low-power tri-axial silicon (MEMS) accelerometer package; the units combined form the flexion angle sensor instrumentation. When static, the sensors register a reading with magnitude $1.0 \mathrm{~g}$ which corresponds to Earth's acceleration due to gravity of $+9.81 \mathrm{~ms}^{-}$ ${ }^{2}$. Using basic trigonometric identities, the angle between the $1.0 \mathrm{~g}$ static acceleration vector and the thigh and shin respectively can be calculated, the sum of which denotes the total angle of knee flexion (Fig. 4). Using two accelerometers also ensures that peak measurement sensitivity is maintained throughout the entire range of motion. The microcontroller uses the axes data to determine the posture of the patient; if the patient has adopted an incorrect posture, is moving, or has incorrectly attached the RFM device, audio and visual notifications are triggered and the event is relayed back to the clinician through the clinician program. This allows unambiguous determination of the knee flexion angle regardless of the overall orientation of the patient, whether they be recumbent, semi-recumbent, standing or at an arbitrary azimuth angle.

The RFM device exploits GSM telephone technology to transmit data via the mobile telephone network to the Internet, and from there to a secure server. The use of a $2 \mathrm{G}$ modem with GPRS is favoured over a $3 \mathrm{G}$ or $4 \mathrm{G}$ modem since it is more economical, offers wider national and global coverage, and typical data transfer rates that permit patient records to be transferred in under one minute [13-15]. Furthermore, backwards compatibility between these standards presents no issues with future technology migration.

During exercise sessions the device samples the axes data at a rate of 20 samples per second and stores each session in flash memory. In the absence of a GSM signal or an external network error during data transmission, the central processing unit buffers the date-time stamped data for 
transmission at the next available opportunity. Before the data are transmitted, they are anonymised via the use of alphanumeric codes associated with each RFM device known to the clinician and basic encryption is performed.

Simplicity and ease of use of the RFM system are primary requirements and as such these characteristics manifest themselves in the aesthetics of the monitoring device. A minimal, selfcontained and intuitive ergonomic is achieved through making the device acutely aware of its handling and usage. Button-less tap-to-start functionality, battery power management, and clear visual and audible feedback indicators are elements of device intelligence which make the system readily deployable to the full age spectrum of TKA patients.

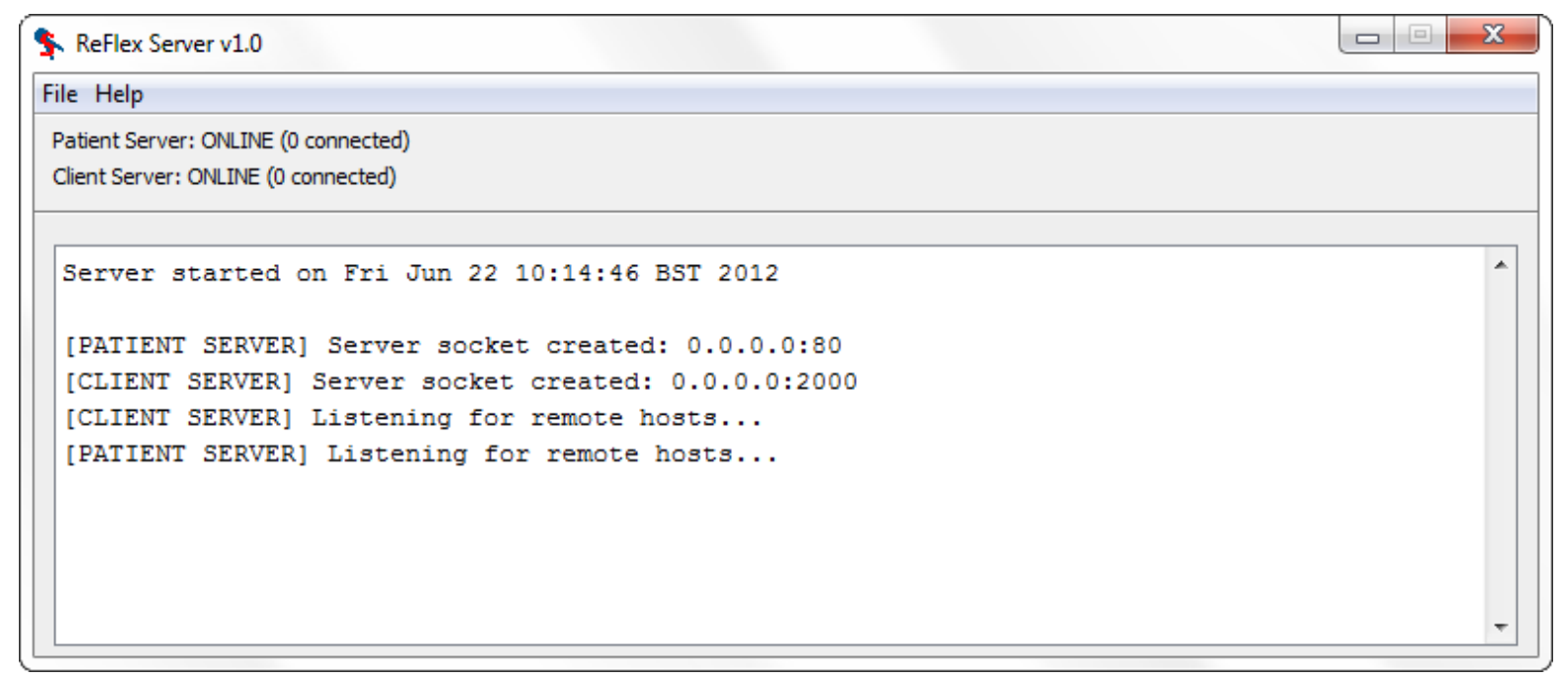

Fig. 5 Hospital-based Java RFM server handles connections as follows: incoming patient exercise data from remote flexion monitoring devices (inbound patient connections), and bi-directional communication with the RFM app that encompasses requests for patient data and transmission of that data (outbound connections to clinician app).

\section{RFM System Server and Clinician App}

The RFM system server enables exercise data to be received and stored securely at the hospital site. Being internet-based, the exercise data are retrievable by the clinician from their computer or tablet from any location or virtual private network with access to the hospital intranet.

Both the server software and the clinician software were developed in the Java programming language which by virtue of being architecture-neutral and portable enables compatibility across a broad spectrum of operating platforms.

The RFM system server operates by listening for requests at two pre-assigned internet addresses corresponding to device-to-server connections and clinician-server connections (Fig. 5). Multiple connections are handled concurrently, thus effectively providing immediate service for any request. Device-to-server connections cause the server to record incoming exercise data to local hard disk 
storage. Clinician-to-server connections initiate the transfer of the stored exercise data to the clinician's computer, where it is processed and can be easily viewed by the clinician.

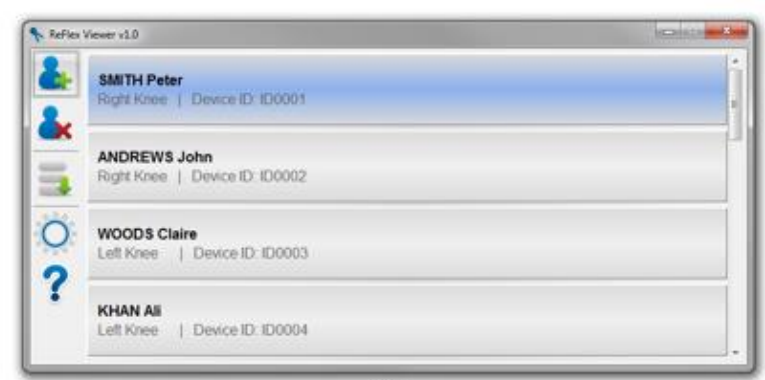

(a)

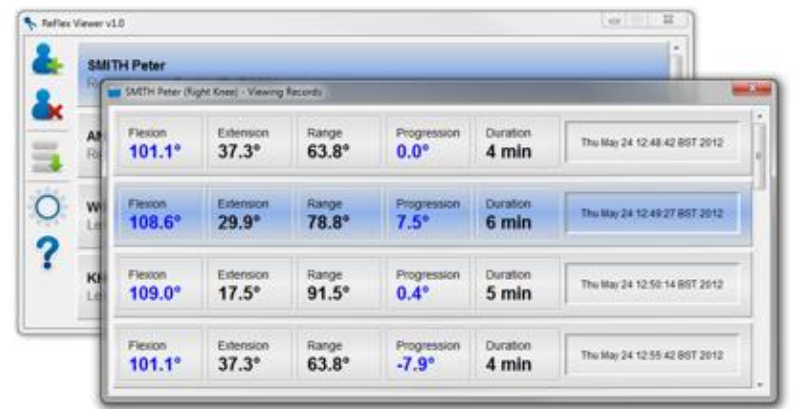

(b)

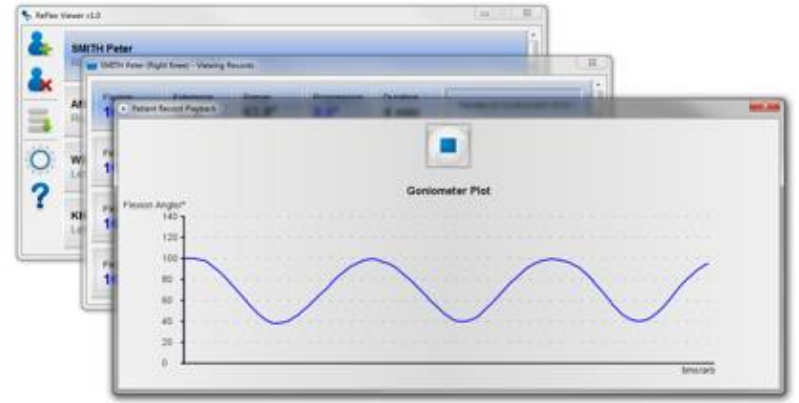

(c)

Fig. 6 Clinician app for analysing exercise data. (a) Graphical user interface lists each patient's name and TKA knee. The icons panel on the left allows the clinician to easily add new patients, delete existing records, and update patient records. (b) Selecting a patient opens a window displaying maximum flexion and extension, progression in maximum flexion since last exercise session, duration of the exercise, and a date-time stamp. The latter two details specifically can be used to ascertain the degree of patient compliance. (c) A visual plot of the patient's flexion motion can also be displayed.

An important theme of this work is convenience for both health services and patients. This is embraced in the clinician end of the RFM system by the use of a clinician program. The clinician program is a portable, web-distributed, and informatively designed application (Fig. 6). A single button click transfers all relevant records from the RFM system server to the clinician's computer, whereat graphing and multiple analysis functions reveal the rate of progression or regression of the patient, thus enabling the clinician to infer the current prognosis. 


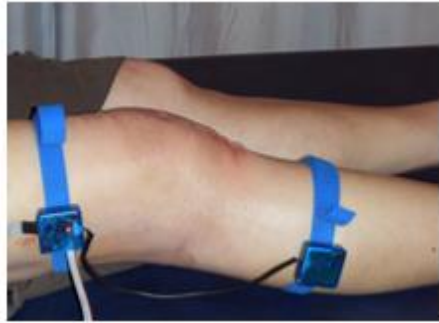

(a)

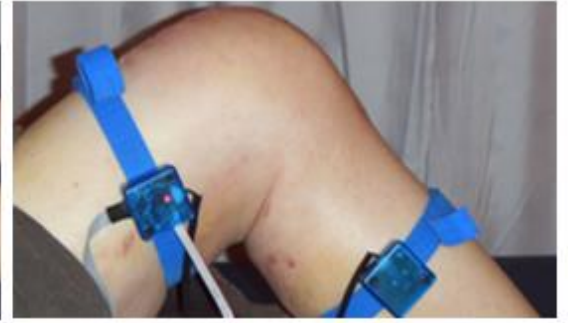

(b)

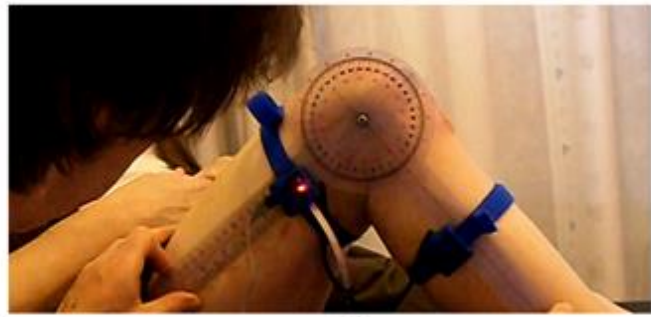

(c)

Fig. 7 An early prototype of the remote flexion monitoring device being tested on a TKA patient (female, right knee). (a) extension, (b) flexion, and (c) to validate device measurements an experienced clinician (MC) measured multiple flexion angles using a goniometer (gold standard method) which were compared against simultaneously acquired measurements from the RFM device.

\section{Conclusion}

A late-stage prototype of a remote flexion monitoring system has been implemented (early device prototype and validation shown in Fig. 7). The system, which comprises two small units attached above and below the knee, records knee flexion data in the domestic environment of the patient in a completely automatic and integrated manner, transmitting the data across the Internet via the GSM mobile network to the clinician's computer. It requires no communication device or internet infrastructure on the part of the patient. Clinical trials of the system are soon to be initiated. Once the clinical effectiveness of the RFM system is validated for TKA patients, other orthopaedic procedures such as hip and shoulder joint replacements, ligament reconstructions and tendon rupture repair may be developed to allow the patient to have rehabilitation monitored at home remotely.

\section{Acknowledgements}

The authors thank the Arthritis Research UK Epidemiology Unit's Research User Group for offering their feedback and constructive criticism on physical and functional aspects of the monitoring device.

The authors also extend their thanks to the Physiotherapy Outpatient Department at Manchester Royal Infirmary for supporting the project by facilitating access to TKA patients attending the Physiotherapy Outpatient Gym.

This study was funded through a Research Councils UK Knowledge Transfer Award (EPSRC grant number KTA-182). 


\section{References}

1. National Joint Registry for England and Wales: 7th Annual Report 2010. Annual Report. September 2010.

2. The Knee: Reilly KA, Beard DJ, Barker KL, Dodd CAF, Price AJ, Murray DW. Efficacy of an accelerated recovery protocol for Oxford unicompartmental knee arthroplasty-a randomised controlled trial. Knee. 2005;12(5):351-7.

3. The Journal of Arthroplasty: Lee RJ, Mears SC. Reducing and Recycling in Joint Arthroplasty. J Arthroplasty. 2012(0).

4. The Journal of Bone and Joint Surgery-American Volume: Kurtz S, Ong K, Lau E, Mowat F, Halpern M. Projections of Primary and Revision Hip and Knee Arthroplasty in the United States from 2005 to 2030. J Bone Joint Surg Am. [Journal]. 2007;89(4).

5. The Journal of Arthroplasty: Pierson JL, Earles DR, Wood K. Brake response time after total knee arthroplasty: When is it safe for patients to drive? J Arthroplasty. 2003;18(7):840-3.

6. The Knee: Sen A, Gocen Z, Unver B, Karatosun V, Gunal I. The frequency of visits by the physiotherapist of patients receiving home-based exercise therapy for knee osteoarthritis. Knee. 2004;11(2):151-3.

7. Rheumatology: Green J, McKenna F, Redfern EJ, Chamberlain MA. Home Exercises are as Effective as Outpatient Hydrotherapy for Osteoarthritis of the Hip. Rheumatology. 1993 September 1, 1993;32(9):812-5.

8. The Journal of Arthroplasty: Edwards JZ, Greene KA, Davis RS, Kovacik MW, Noe DA, Askew MJ. Measuring flexion in knee arthroplasty patients. J Arthroplasty. 2004;19(3):36972.

9. Kemp T. EDC: Deploying devices in clinical trials. Hertfordshire: Clinical Discovery; 2007 [updated 20 September 2007; cited 2012 03/07/2012]; Available from: http://www.clinicaldiscovery.com/readArticle.aspx?articleId=59.

10. Controlled Clinical Trials: Lauritsen K, Degl' Innocenti A, Hendel L, Præst J, Lytje MF, Clemmensen-Rotne K, et al. Symptom recording in a randomised clinical trial: paper diaries vs. electronic or telephone data capture. Control Clin Trials. 2004;25(6):585-97.

11. Controlled Clinical Trials: Stone AA, Shiffman S, Schwartz JE, Broderick JE, Hufford MR. Patient compliance with paper and electronic diaries. Control Clin Trials. 2003;24(2):182-99.

12. Telemedicine and e-Health: Stoepel C, Boland J, Busca R, Saal G, Oliveira M. Usefulness of Remote Monitoring in Cardiac Implantable Device Follow-Up. Telemed J E Health: 2009 2009/12/01;15(10):1026-30.

13. Ofcom. Communications Market Report: UK. Research Document: Ofcom 20114 August 2011. 
14. Ofcom. International Communications Market Report 2011. Research Document: Ofcom 201114 December 2011.

15. Telemedicine and e-Health: Pappas G. Planning for Internet Connectivity in Remote Patient Monitoring. Telemed J E Health: 2010 2010/06/01;16(5):639-41.

\section{Figure captions}

Fig. 1 Remote flexion monitoring system architecture: data flow from remotely-located patients to the clinician app is shown

Fig. 2 Schematic depicting the main components of the RFM device electronics which comprise a master and slave unit

Fig. 3 RFM device master unit in which the 2.5G GSM modem is visible with a size comparison

Fig. 4 Trigonometric identities used for calculating the angle of knee flexion using two independent accelerometers; one strapped to the thigh, and the second strapped to the shin.

Fig. 5 Hospital-based Java RFM server handles connections as follows: incoming patient exercise data from remote flexion monitoring devices (inbound patient connections), and bi-directional communication with the RFM app that encompasses requests for patient data and transmission of that data (outbound connections to clinician app).

Fig. 6 Clinician app for analysing exercise data. (a) Graphical user interface lists each patient's name and TKA knee. The icons panel on the left allows the clinician to easily add new patients, delete existing records, and update patient records. (b) Selecting a patient opens a window displaying maximum flexion and extension, progression in maximum flexion since last exercise session, duration of the exercise, and a date-time stamp. The latter two details specifically can be used to ascertain the degree of patient compliance. (c) A visual plot of the patient's flexion motion can also be displayed.

Fig. 7 An early prototype of the remote flexion monitoring device being tested on a TKA patient (female, right knee). (a) extension, (b) flexion, and (c) to validate device measurements an experienced clinician (MC) measured multiple flexion angles using a goniometer (gold standard method) which were compared against simultaneously acquired measurements from the RFM device. 